\title{
A High-order Implicit Difference Method for the One-dimensional Convection Diffusion Equation
}

\author{
Hua Huang \\ School of Mechanical Engineering, YanCheng Technician College \\ Yancheng 224000, JiangSu, China \\ Tel: 86-158-5108-7750 E-mail: hhuaycjs@163.com \\ Suzhen Huang \\ Department of Basic Science, Yancheng Institute of Technology \\ Yancheng 224051, China \\ Tel: 86-158-6197-2996 E-mail: hszhszhsz2002@yahoo.com.cn
}

Received: March 1, 2011 Accepted: March 17, 2011 doi:10.5539/jmr.v3n3p135

\begin{abstract}
Based on the exponent transform to eliminate the "convection item" in the equation and the fourth-order compact difference formulas for the first and second derivatives, two chasses of new implicit difference schemes are proposed for solving the one-dimensional convection-diffusion equation. The methods are of order $O\left(\tau^{2}+h^{4}\right)$ and $O\left(\tau^{4}+h^{4}\right)$ respectively. The former is proved to be unconditionally stable while the later is unconditionally unstable by Fourier analysis. The result of numerical experiment shows that the $O\left(\tau^{2}+h^{4}\right)$ scheme is an effective difference scheme to solve the convection diffusion problem.
\end{abstract}

Keywords: Convection diffusion equation, Compact implicit difference, High-order, Unconditionally stable

\section{Introduction}

For one-dimensional convection diffusion problem,

$\begin{cases}\frac{\partial u}{\partial t}+k \frac{\partial u}{\partial x}=d \frac{\partial^{2} u}{\partial x^{2}} & 0<x<1,0<t \leq T \\ u(x, 0)=f(x) & 0 \leq x \leq 1 \\ u(0, t)=g_{0}(t), u(1, t)=g_{1}(t) & 0<t \leq T\end{cases}$

here, $f, g_{0}$ and $g_{1}$ are known functions, and $u$ is unknown function, $\alpha$ and $\beta$ are constants greater than 0 .

The numerical solution of the convection diffusion equation is the important part in the numerical math, and in tens of years, it has been studied and applied further (Douglas J, 1982, P.871-885 \& Lu, 1998, P.161-167 \& Wang, 2002, P.194199 \& Lu, 2002, P.35-37 \& Qin, 2003, P.25-27 \& Huang, 2005, P.38-41 \& Mehdi Dehghan, 2004, P.307-319 \& Mehdi Dehghan, 2004, P.5-19). But the standard difference method or the finite element method often fail to this problem, and the essential cause is the existence of the "convection item". The exponential transform is used in this article to transform the equation and eliminate the "convection item" in the equation, and the transformed equation is diffusion equation, because it has many effective numerical solutions (Ge, 2005, P.107-110). Based on that, the $O\left(\tau^{2}+h^{4}\right)$ difference scheme and the $O\left(\tau^{4}+h^{4}\right)$ difference scheme are constructed to solve the one-dimensional convection diffusion equation. The Fourier method is used to analyze the stability of the difference scheme in this article, and the accuracy and reliability of the method are tested by the numerical example.

\section{Exponential transform}

To eliminate the convection item in the equation, supposed that $u=e^{\alpha x+\beta t} v$, so $\frac{\partial u}{\partial t}=\left(\beta v+\frac{\partial v}{\partial t}\right) e^{\alpha x+\beta t}, \frac{\partial u}{\partial x}=\left(\alpha v+\frac{\partial v}{\partial x}\right) e^{\alpha x+\beta t}$, and $\frac{\partial^{2} u}{\partial x^{2}}=\left(\alpha^{2} v+2 \alpha \frac{\partial v}{\partial x}+\frac{\partial^{2} v}{\partial x^{2}}\right) e^{\alpha x+\beta t}$.

Substituting above derivatives into the formula (1), so $\frac{\partial v}{\partial t}+(k-2 \alpha d) \frac{\partial v}{\partial x}=d \frac{\partial^{2} v}{\partial x^{2}}+\left(d \alpha^{2}-k \alpha-\beta\right) v$.

And if $\left\{\begin{array}{l}k-2 \alpha d=0 \\ d \alpha^{2}-k \alpha-\beta=0\end{array}\right.$, so when $\left\{\begin{array}{l}\alpha=\frac{k}{2 d} \\ \beta=-\frac{k^{2}}{4 d}\end{array}\right.$, the formula (1) could be transformed as following form.

$$
\frac{\partial v}{\partial t}=d \frac{\partial^{2} v}{\partial x^{2}}
$$




\section{Establishment of the difference scheme}

First, establish the difference grid. Take the space step length $h>0$, the node $x_{j}=j h, j=0,1,2, \cdots, M=[1 / h]$, and the time step length $\tau>0$, the node $t_{n}=n \tau, n=0,1,2, \cdots, N=[T / \tau]$. And for simplifying the discussion, supposed that $k>0, d>0$.

$3.1 O\left(\tau^{2}+h^{4}\right)$ scheme

To keep the network frame of two layers and three points in the scheme, consider the value of the formula (4) at the time of $n+\frac{1}{2}$, so

$$
\left[\frac{\partial v}{\partial t}\right]_{j}^{n+\frac{1}{2}}=d\left[\frac{\partial^{2} v}{\partial x^{2}}\right]_{j}^{n+\frac{1}{2}}
$$

In space, the four-order compact difference is used to approach the formula,

$$
\left[\frac{\partial^{2} v}{\partial x^{2}}\right]_{j}=\left[1+\frac{h^{2}}{12} \delta_{x}^{2}\right]^{-1} \delta_{x}^{2} v_{j}+O\left(h^{4}\right)
$$

where,

$$
\delta_{x}^{2} v_{j}=\frac{v_{j+1}-2 v_{j}+v_{j-1}}{h^{2}}
$$

And substitute $v_{j}^{n+\frac{1}{2}}$ by the arithmetic mean value of $v_{j}^{n}$ and $v_{j}^{n+1}$, and take the central difference of the time item of $\left[\frac{\partial v}{\partial t}\right]_{j}^{n+\frac{1}{2}}$,

$$
\frac{v_{j}^{n+1}-v_{j}^{n}}{\tau}=\frac{d}{2}\left[1+\frac{h^{2}}{12} \delta_{x}^{2}\right]^{-1} \delta_{x}^{2}\left(v_{j}^{n}+v_{j}^{n+1}\right)
$$

So,

$$
\left[1+\frac{h^{2}}{12} \delta_{x}^{2}\right]\left(v_{j}^{n+1}-v_{j}^{n}\right)=\frac{d \tau}{2} \delta_{x}^{2}\left(v_{j}^{n}+v_{j}^{n+1}\right)
$$

Unfold the formula (20) according to the form of the formula (7), and suppose $r=d \frac{\tau}{h^{2}}$, so

$$
(10+12 r) v_{j}^{n+1}+(1-6 r)\left(v_{j+1}^{n+1}+v_{j-1}^{n+1}\right)=(10-12 r) v_{j}^{n}+(1+6 r)\left(v_{j+1}^{n}+v_{j-1}^{n}\right)
$$

The difference scheme of the formula (1) can be obtained by the inverse transformation, $V=e^{-(\alpha x+\beta t)} U$, so

$$
\begin{aligned}
& (10+12 r) u_{j}^{n+1}+(1-6 r)\left(e^{-\alpha h} u_{j+1}^{n+1}+e^{\alpha h} u_{j-1}^{n+1}\right) \\
& =e^{\beta \tau}\left[(10-12 r) u_{j}^{n}+(1+6 r)\left(e^{-\alpha h} u_{j+1}^{n}+e^{\alpha h} u_{j-1}^{n}\right)\right]
\end{aligned}
$$

it has the compact implicit scheme with two layers and three points, and from the deduction process, its truncation error is $O\left(\tau^{2}+h^{4}\right)$.

$3.2 O\left(\tau^{4}+h^{4}\right)$ scheme

To enhance the time order to four-order, use the four-order formula to the time derivative item.

$$
\left[\frac{\partial v}{\partial t}\right]^{n}=\left[1+\frac{\tau^{2}}{6} \delta_{t}^{2}\right]^{-1} \delta_{t} v^{n}+O\left(\tau^{4}\right)
$$

Where,

$$
\left.\begin{array}{l}
\delta_{t} v^{n}=\frac{v^{n+1}-v^{n-1}}{2 \tau} \\
\delta_{t}^{2} v^{n}=\frac{v^{n+1}-2 v_{n}+v^{n-1}}{\tau^{2}}
\end{array}\right\}
$$

For the time derivative item, the four-order compact difference is still used to approach the formula (6), and considering the value of the formula (4) at the time of $n$, 


$$
\left[1+\frac{\tau^{2}}{6} \delta_{t}^{2}\right]^{-1} \delta_{t} v_{j}^{n}=d\left[1+\frac{h^{2}}{12} \delta_{x}^{2}\right]^{-1} \delta_{x}^{2} v_{j}^{n}
$$

i.e.,

$$
\delta_{t}\left[1+\frac{h^{2}}{12} \delta_{x}^{2}\right] v_{j}^{n}=d \delta_{x}^{2}\left[1+\frac{\tau^{2}}{6} \delta_{t}^{2}\right] v_{j}^{n}
$$

Substitute the formula (7) and the formula (13) into the formula (19), and supposed that $r=d \frac{\tau}{h^{2}}$, so

$$
\begin{aligned}
& (5+4 r) v_{j}^{n+1}+\left(\frac{1}{2}-2 r\right)\left(v_{j+1}^{n+1}+v_{j-1}^{n+1}\right) \\
& \quad=-16 r v_{j}^{n}+8 r\left(v_{j+1}^{n}+v_{j-1}^{n}\right)+(5-4 r) v_{j}^{n-1}+\left(\frac{1}{2}+2 r\right)\left(v_{j+1}^{n-1}+v_{j-1}^{n-1}\right)
\end{aligned}
$$

The difference scheme of the formula (1) can be obtained by the inverse transformation, $V=e^{-(\alpha x+\beta t)} U$, so

$$
\begin{aligned}
e^{-\beta \tau}[(5 & \left.+4 r) u_{j}^{n+1}+\left(\frac{1}{2}-2 r\right)\left(e^{-\alpha h} u_{j+1}^{n+1}+e^{\alpha h} u_{j-1}^{n+1}\right)\right] \\
& =-16 r u_{j}^{n}+8 r\left(e^{-\alpha h} u_{j+1}^{n}+e^{\alpha h} u_{j-1}^{n}\right) \\
& +e^{\beta \tau}\left[(5-4 r) u_{j}^{n-1}+\left(\frac{1}{2}+2 r\right)\left(e^{-\alpha h} u_{j+1}^{n-1}+e^{\alpha h} u_{j-1}^{n-1}\right)\right]
\end{aligned}
$$

it has the compact implicit scheme with two layers and three points, and from the deduction process, its truncation error is $O\left(\tau^{4}+h^{4}\right)$.

\section{Stability analysis}

Supposed that the boundary conditions are satisfied exactly, and to prove the stability, the Lemma is introduced.

Lemma (Lu, 1987): The sufficient and necessary condition that the module of the real coefficient two-order equation $x^{2}-b x-c=0$ is less than 1 is $|b| \leq 1-c \leq 2$.

4.1 Stability analysis of $O\left(\tau^{2}+h^{4}\right)$ scheme

The Fourier method is used to prove the stability of the difference scheme (11). It is easy to solve that the growth factor is

$$
G=\frac{10+2 \cos \sigma h-12 r(1-\cos \sigma h)}{10+2 \cos \sigma h+12 r(1-\cos \sigma h)}
$$

and because $1-\cos \sigma h \geq 0$, so $|G| \leq 1$, i.e. the formula (1) is unconditionally stable.

4.2 Stability analysis of $O\left(\tau^{4}+h^{4}\right)$ scheme

The Fourier method is used to prove the stability of the difference scheme (17). It is easy to solve that the growth matrix is

$$
G=\left[\begin{array}{ll}
\frac{-16 r(1-\cos \sigma h)}{5+\cos \sigma h+4 r(1-\cos \sigma h)} & 5+\cos \sigma h-4 r(1-\cos \sigma h) \\
\frac{1}{5+\cos \sigma h+4 r(1-\cos \sigma h)} & 0
\end{array}\right]
$$

and its characteristic equation is

$$
x^{2}+\frac{16 r(1-\cos \sigma h)}{5+\cos \sigma h+4 r(1-\cos \sigma h)} x-\frac{5+\cos \sigma h-4 r(1-\cos \sigma h)}{5+\cos \sigma h+4 r(1-\cos \sigma h)}=0
$$

so

$$
|b|+c=\frac{5+\cos \sigma h+12 r(1-\cos \sigma h)}{5+\cos \sigma h+4 r(1-\cos \sigma h)}
$$

and obviously, $|b|+c>1$, so from the Lemma, the scheme (17) is unstable without preconditions.

\section{Numerical experiment}

For the problem with the steady state solution, 


$$
\left\{\begin{array}{lr}
\frac{\partial u}{\partial t}+k \frac{\partial u}{\partial x}=d \frac{\partial^{2} u}{\partial x^{2}} & (0<x<1,0<t<T, d>0) \\
u(x, 0)=0 & (0<x<1), \\
u(0, t)=0, u(1, t)=1 & (0<t<T) .
\end{array}\right.
$$

its exact solution is (D.J.Evans, 1985, P.145-154),

$$
u(x, t)=\frac{e^{k x / d}-1}{e^{k / d}-1}+\sum_{m=1}^{\infty} \frac{(-1)^{m} m \pi}{(m \pi)^{2}+\left(\frac{k}{2 d}\right)^{2}} e^{k(x-1) / 2 d} \sin (m \pi x) e^{-\left[(m \pi)^{2} d+k^{2} / 4 d\right] t}
$$

By respectively using the $O\left(\tau^{2}+h^{4}\right)$ scheme and the $O\left(\tau^{4}+h^{4}\right)$ scheme, and taking $k=1.0, d=1.0$ and $h=0.02$, the numerical result when $t=0.4$ is computed when $\tau=0.004$ and $\tau=0.0004$, and the results are respectively seen in Table 1 and Table 2.

The numerical result shows that solving the non-convection dominant diffusion problem by the scheme of $O\left(\tau^{2}+h^{4}\right)$ could obtain small numerical diffusion, few numerical vibration, and highorder. At the same time, because each time layer only has three grid points, so the difference equation tri-diagonal equation, and it can be solved by the chase-after method directly with less computation quantity and without iteration. When $\tau=O\left(h^{2}\right)$, the computation achieves the fourorder precision in space, and it is stable for any big grid ratio $r$. The $O\left(\tau^{4}+h^{4}\right)$ scheme is unstable without preconditions, so it cannot be used in practical computation.

\section{References}

D.J.Evans \& A.R.Abdullah. (1985). A New Explicit Method for the Diffusion -convection Equations. Comp and Math with Appl, No.11. P.145-154.

Douglas J \& Russell T F. (1982). Numerical methods for convection-dominated diffusion problem based on combining the method of characteristics with finite element or finite difference procedures. SIAM J Numer Anal, No.19(5). P.871-885.

Ge, Yongbin, Tian, Zhenfu, Zhan, Yong \& Wu, Wenquan. (2005). High-order Implicit Difference Method for the Diffusion Equation. Journal of University of Shanghai for Science and Technology, No.27(2). P.107-110.

Huang, Suzhen \& Zhang, Luming. (2005). A Class of High Accuracy Characteristic Difference Method for ConvectionDiffusion Equations. Journal of Nanjing Normal University (Natural Science), No.28(2). P.38-41.

Lu, Jinfu, Du, Zhengping \& Liu, Xiaoyu. (2002). Characteristic Difference Method for the Convection-dominated Diffusion Problems. Journal of Tsinghua University (Science and Technology), No.42(8). P.35-37.

Lu, Jinfu \& Guan, Ye. (1987). Numerical Methods for PDE. Beijing: Tsinghua University Press.

Lu, Jinfu, Zhang, Baolin \& Xu, Tao. (1998). Explicit-implicit Method to Solve the Alternation Segmentation of the Convection Diffusion Equation. Journal on Numerical Methods and Computer Application, No.19(3). P.161-167.

Mehdi Dehghan. (2004). Numerical solution of the three-dimensional advection-diffusion equation, Applied Mathematics and Computation, No.150 (1). P.5-19.

Mehdi Dehghan. (2004). Weighted finite difference techniques for the one-dimensional advection-diffusion equation, Applied Mathematics and Computation, No.147 (2). P.307-319.

Qin, Xinqiang \& Li, Qiufang. (2003). A New Kind of Characteristic-Difference Algorithm for Convection-Diffusion Equations. Journal of Xi'an University of Technology, No.19(2). P.25-27.

Wang, Wenqia. (2002). A Class Alternating Group Explicit Scheme for Solving Diffusion Equation and Parallel Computing. Journal of Shandong University (Natural Science Edition), No.37(3). P.194-199. 
Table 1. Numerical result when $k=1.0, \mathrm{~d}=1.0, \tau=0.004, h=0.02, t=0.4$

\begin{tabular}{|l|l|l|l|l|l|}
\hline \multirow{2}{*}{$j\left(x_{j}\right)$} & \multicolumn{2}{|l|}{$O\left(\tau^{2}+h^{4}\right)$ scheme } & \multirow{2}{*}{$O\left(\tau^{2}+h^{4}\right)$ scheme } & \multirow{2}{*}{ Exact solution } \\
\cline { 2 - 3 } & $\begin{array}{l}\text { Numerical solu- } \\
\text { tion }\end{array}$ & Absolute error & Relative error & & \\
\hline 5 & 0.059072 & 0.001067 & 0.017746 & & 0.060139 \\
\hline 10 & 0.124582 & 0.002138 & 0.016875 & 0.12672 \\
\hline 15 & 0.197432 & 0.003088 & 0.015401 & 0.20052 \\
\hline 20 & 0.278596 & 0.003814 & 0.013506 & \\
\hline 25 & 0.369101 & 0.004219 & 0.011300 & \multirow{3}{*}{ Unstable without preconditions } & 0.28241 \\
\hline 30 & 0.470016 & 0.004214 & 0.008886 & 0.47423 \\
\hline 35 & 0.582434 & 0.003776 & 0.006441 & & 0.58621 \\
\hline 40 & 0.707472 & 0.002878 & 0.004051 & & 0.71035 \\
\hline 45 & 0.846260 & 0.001600 & 0.001887 & & 0.84786 \\
\hline
\end{tabular}

Table 2. Numerical result when $k=1.0, \mathrm{~d}=1.0, \tau=0.0004, h=0.02, t=0.4$

\begin{tabular}{|c|c|c|c|c|c|}
\hline$j\left(x_{j}\right)$ & \multicolumn{3}{|l|}{$O\left(\tau^{2}+h^{4}\right)$ scheme } & \multirow{2}{*}{$O\left(\tau^{2}+h^{4}\right)$ scheme } & \multirow{2}{*}{ Exact solution } \\
\hline & Numerical solution & Absolute error & Relative error & & \\
\hline 5 & 0.059072 & 0.001067 & 0.017747 & \multirow{9}{*}{ Unstable without preconditions } & 0.060139 \\
\hline 10 & 0.124581 & 0.002139 & 0.016876 & & 0.12672 \\
\hline 15 & 0.197431 & 0.003089 & 0.015403 & & 0.20052 \\
\hline 20 & 0.278595 & 0.003815 & 0.013508 & & 0.28241 \\
\hline 25 & 0.369101 & 0.004219 & 0.011302 & & 0.37332 \\
\hline 30 & 0.470016 & 0.004214 & 0.008887 & & 0.47423 \\
\hline 35 & 0.582434 & 0.003776 & 0.006441 & & 0.58621 \\
\hline 40 & 0.707473 & 0.002877 & 0.004051 & & 0.71035 \\
\hline 45 & 0.846270 & 0.001590 & 0.001876 & & 0.84786 \\
\hline
\end{tabular}

